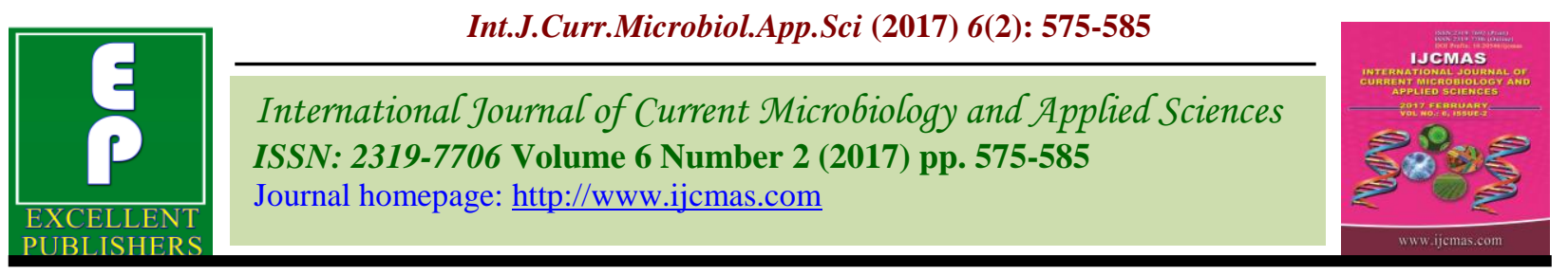

Original Research Article

http://dx.doi.org/10.20546/ijcmas.2017.602.065

\title{
Evaluation of Sex Pheromone Trap Models for Monitoring of Shoot and Fruit Borer, Leucinodes orbonalis Gueene. in Brinjal
}

\author{
M. Aravinda ${ }^{1}$, S.S. Udikeri ${ }^{2} *$ and S.S. Karabhantanal ${ }^{1}$ \\ ${ }^{1}$ Department of Agricultural Entomology, College of Agriculture, Vijayapur, India \\ ${ }^{2}$ University of Agricultural Sciences, Dharwad - 580 005, India \\ *Corresponding author
}

\section{A B S T R A C T}

\begin{tabular}{|l|}
\hline Ke y w o r d s \\
Brinjal, Shoot and \\
fruit borer, \\
Leucinodes \\
orbonalis, \\
Pheromone traps.
\end{tabular}

\section{Introduction}

Brinjal fruit (unripe) is primarily consumed as cooked vegetable in various ways and dried plants are used as fuel in rural areas. Brinjal fruits are widely used in various culinary preparations Viz., sliced bhaji, stuffed curry, bertha, chutney, vangibath, pickles etc. In India the area under brinjal cultivation was estimated to be 0.72 mha with total production of $13.56 \mathrm{mt}$ and the productivity of $19.10 \mathrm{t} \mathrm{ha}^{-1}$. In Karnataka, it is grown in an area of $0.0158 \mathrm{~m}$ ha with a total production of $0.4025 \mathrm{mt}$ and the productivity is $25.40 \mathrm{t} \mathrm{ha}^{-1}$ (Anon, 2014a). The yields at state and national level are very low compared to their potential productivity. There are several factors responsible for low yield, among them insect pests are major once. As many as 70 insect pests have been reported on brinjal, Among them, brinjal shoot and fruit borer (BSFB), (Leucinodes orbonalis Guenee: Pyralidae: Lepidoptera) is considered as a major and most destructive pest. In the early stage of the crop, larva bores into the shoots resulting in drooping, withering and drying of the affected shoots. During the reproductive stage, tiny shiny larva bores into the flower buds and fruits. The bore hole is invariably plugged with excreta. It inflicts sizeable damage up to 80 per cent in terms of fruit and content of vitamin-C (Ram Prasad Mainali, 
2014). The yield loss could be as high as 70 per cent (Dhandapani et al., 2003) and may go up to 90 per cent in India (Kalloo, 1988). Therefore an attempt has been made to evaluate different types of pheromone trap models for monitoring Leucinodes orbonalis to guide plant protection schedule or for mass trapping exercise.

\section{Materials and Methods}

Field investigation was carried out on brinjal to assess the performance of different monitoring traps of shoot and fruit borer, Leucinodes orbonalis Guene. The experiment was conducted in irrigated condition during kharif 2014-15 at Hittinahalli and Jumanal villages of Vijayapur district of Karnataka state. The seedlings of Mahyco Super -10 were transplanted in a plot size of $10 \times 10 \mathrm{~m}^{2}$ and crop was raised as per the recommended package of practice (Anon, 2014b). Sucking pests were managed with uniform treatments across experiment. Application of Rynaxypyr (Coragen) was given as curative measure to avoid possible complete loss due to pest as the crop was raised for commercial purpose. The experiment was laid out in randomized block design with following six treatments and four replications.

\section{Treatment details}

$\mathbf{T}_{\mathbf{1}}$-Water trap (PCI Commercial Model)

$\mathbf{T}_{\mathbf{2}}$-Sleeve trap (PCI Commercial Model)

$\mathbf{T}_{\mathbf{3}}$-Delta trap (PCI Commercial Model)

$\mathbf{T}_{4}$-Bottle trap (Locally prepared)

$\mathbf{T}_{5}$-Can trap (Locally prepared)

$\mathbf{T}_{\mathbf{6}}$ - Untreated control

Soon after transplanting the seedling into field, different models of traps Viz., commercial models of water trap, sleeve trap and delta trap and locally prepared bottle and can traps were installed in field at $10 \mathrm{~m} \times 10 \mathrm{~m}$ distance. 1.0 L empty drinking water bottles and 5.0 L empty oil cans were used to develop local type traps. Water bottle traps had a rupee coin wide hole and can type trap had a $5.0 \mathrm{~cm}^{2}$ hole provision for entry of moths. The BSFB specific PCI commercial lures were used in the study which has been changed once in 15 days after installation. Traps were about a feet above the crop canopy. The observations were recorded at five days interval on number of moths caught in each trap after one week of installation. The moths were disposed off after every observation. Plants from ten feet radius from traps were selected from each field for shoot damage observation. The extent of damage was expressed in percentage using the formula given below.

Per cent shoot infestation $=\frac{\text { Number of infested shoots }}{\text { Total number of shoots }} \times 100$

Similarly, the per cent fruit infestation was calculated by counting the number of infested

fruits and total number of fruits by using the formula mentioned below.

Per cent fruit infestation $=\frac{\text { Number of infested fruit }}{\text { Total number of fruit }} \times 100$

Total number of fruit

The fruit yield was recorded as per plot basis basis. from 30 pickings were converted to hectare 


\section{Results and Discussion}

Data on the number of moth catches in different traps are presented in table 1. It was found that water trap was significantly superior over the other treatments in attracting moths with a seasonal mean catch of 3.08/trap / 5 days). It was superior over rest of the models except the can trap (2.03 moths /trap). However, the latter treatment was on par with bottle trap (1.06 moths / trap) followed by sleeve trap (0.96 moths / trap) and delta trap (0.72 moths / trap). Since the lure source and changing frequency was not varying among the treatments, the difference in turns of moths catches was exclusively the impact of trapping device structure or model. Similar to present finding Alam et al., (2003) have observed trap model dependent variation in behavioural response of moths.

During the investigation, water trap was found to be excellent in trapping the moths compared to other traps. This may be due to the open space in water trap where the moths come and hit the sides of the trap and fall into the water containing insecticides. In case of water trap, the chance of escape of moth was very less unlike other traps. The present finding is in accordance with Andagopal et al., (2011) who reported that the Wota-T trap as best one. Rajneesh, (2006) reported that the number of moths caught in water trap was found to be highly significant in catching the more number of moths at $1^{\text {st }}$ week after installation.

Data on the per cent shoot damage due shoot and fruit damage is presented in table 2 . Significantly mean seasonal lowest shoot damage was recorded in water trap $(5.48 \%)$ and was on par with can trap $(6.56 \%)$ followed by bottle trap (7.22 \%), sleeve trap $(8.24 \%)$ and delta trap $(9.16 \%)$. On the contrary, the highest per cent shoot damage was recorded in control $(9.24 \%)$. The reduction in shoot damage in water trap and can trap may be due to more number of moths trapped in these traps which in turn checks the oviposition by the moths. The present findings are in conformity with Chakraborthi (2001) who reported that, the integrated approach including pheromones in the management of brinjal shoot and fruit borer was found highly effective in minimizing the shoot and fruit borer infestation. Similarly, Alam et al., (2003) also stated that removal of damaged shoots and use of pheromone traps resulted in a significant reduction in further development into adults in subsequent generations and reduced the brinjal shoot and fruit infestation.

Data on the fruit damage are presented in table 3 . The average lowest fruit damage was recorded in water trap treatment $(15.03 \%)$ and was on par with locally prepared can trap $(17.83 \%)$. Fruit damage recorded in control $(26.25 \%)$ was significantly highest among treatments. The total highest fruit yield per plot was significantly highest in water trap $489.0 \mathrm{q}$ followed by can trap $\left(416.0 \mathrm{q} \mathrm{ha}^{-1}\right)$. $364.0 \mathrm{q}, 319.0 \mathrm{q}, 278.0 \mathrm{q}$ fruit yield per ha were recorded in bottle trap, sleeve trap and delta trap, respectively and these treatments did not show any superiority over control plot $\left(271.0 \mathrm{q} \mathrm{ha} \mathrm{h}^{-1}\right)$ (table 4). The highest marketable yield obtained in water trap treatment followed by can trap over control was due to reduced pest incidence during the experiment period. The present findings are in conformity with Chakraborthi (2001) reported that the integrated approach including phermones in the management of brinjal shoot and fruit borer was found highly effective and reduced shoot and fruit infestation and recorded higher yield.

The present investigation was carried out under protected situation with the application of insecticides, removal of affected shoots and evaluation of pheromone traps. 
Table.1 Evaluation of different sex pheromone trap models for L. orbonalis monitoring in brinjal crop

\begin{tabular}{|c|c|c|c|c|c|c|c|c|c|c|c|c|c|c|c|}
\hline \multirow[b]{2}{*}{ Treatment } & \multicolumn{15}{|c|}{ Number of moths caught/trap(in five days) } \\
\hline & $\begin{array}{c}5 \\
\text { DAIT }\end{array}$ & $\begin{array}{c}10 \\
\text { DAIT }\end{array}$ & $\begin{array}{c}15 \\
\text { DAIT }\end{array}$ & $\begin{array}{c}20 \\
\text { DAIT }\end{array}$ & $\begin{array}{c}25 \\
\text { DAIT }\end{array}$ & $\begin{array}{c}30 \\
\text { DAIT }\end{array}$ & $\begin{array}{c}35 \\
\text { DAIT }\end{array}$ & $\begin{array}{c}40 \\
\text { DAIT }\end{array}$ & $\begin{array}{c}45 \\
\text { DAIT }\end{array}$ & $\begin{array}{c}50 \\
\text { DAIT }\end{array}$ & $\begin{array}{c}55 \\
\text { DAIT }\end{array}$ & $\begin{array}{c}60 \\
\text { DAIT }\end{array}$ & $\begin{array}{c}65 \\
\text { DAIT }\end{array}$ & $\begin{array}{c}70 \\
\text { DAIT }\end{array}$ & $\begin{array}{c}75 \\
\text { DAIT }\end{array}$ \\
\hline $\begin{array}{c}\mathrm{T} 1 \\
\text { Water trap }\end{array}$ & $\begin{array}{c}6.50 \\
(2.74)^{\mathrm{a}}\end{array}$ & $\begin{array}{c}6.25 \\
(2.69)^{\mathrm{a}}\end{array}$ & $\begin{array}{c}4.00 \\
(2.23)^{\mathrm{a}}\end{array}$ & $\begin{array}{c}8.00 \\
(2.99)^{\mathrm{a}}\end{array}$ & $\begin{array}{c}3.50 \\
(2.12)^{\mathrm{a}}\end{array}$ & $\begin{array}{c}3.00 \\
(2.00)^{\mathrm{a}}\end{array}$ & $\begin{array}{c}1.75 \\
(1.64)^{\mathrm{a}}\end{array}$ & $\begin{array}{c}3.50 \\
(2.12)^{\mathrm{a}}\end{array}$ & $\begin{array}{c}3.25 \\
(2.06)^{\mathrm{a}}\end{array}$ & $\begin{array}{c}2.50 \\
(1.85)^{\mathrm{a}}\end{array}$ & $\begin{array}{c}2.75 \\
(1.93)^{\mathrm{a}}\end{array}$ & $\begin{array}{c}2.25 \\
(1.80)^{\mathrm{a}}\end{array}$ & $\begin{array}{c}2.25 \\
(1.79)^{\mathrm{a}}\end{array}$ & $\begin{array}{c}2.25 \\
(1.80)^{\mathrm{a}}\end{array}$ & $\begin{array}{c}2.50 \\
(1.85)^{\mathrm{a}}\end{array}$ \\
\hline $\begin{array}{c}\mathrm{T} 2 \\
\text { Sleeve trap }\end{array}$ & $\begin{array}{c}2.00 \\
(1.72)^{b}\end{array}$ & $\begin{array}{c}1.50 \\
(1.57)^{\mathrm{c}}\end{array}$ & $\begin{array}{c}1.00 \\
(1.41)^{\mathrm{b}}\end{array}$ & $\begin{array}{c}2.00 \\
(1.73)^{\mathrm{c}}\end{array}$ & $\begin{array}{c}1.00 \\
(1.41)^{\mathrm{c}}\end{array}$ & $\begin{array}{c}1.75 \\
(1.65)^{\mathrm{b}}\end{array}$ & $\begin{array}{c}0.75 \\
(1.31)^{\mathrm{bc}}\end{array}$ & $\begin{array}{c}0.75 \\
(1.31)^{\mathrm{b}}\end{array}$ & $\begin{array}{c}1.75 \\
(1.64)^{\mathrm{b}}\end{array}$ & $\begin{array}{c}1.25 \\
(1.46)^{\mathrm{ab}}\end{array}$ & $\begin{array}{c}0.75 \\
(1.31)^{\mathrm{b}}\end{array}$ & $\begin{array}{c}1.25 \\
(1.49)^{\mathrm{b}}\end{array}$ & $\begin{array}{c}1.50 \\
(1.57)^{\mathrm{b}}\end{array}$ & $\begin{array}{c}1.00 \\
(1.41)^{\mathrm{b}}\end{array}$ & $\begin{array}{c}1.00 \\
(1.41)^{\mathrm{c}}\end{array}$ \\
\hline $\begin{array}{c}\text { T3 } \\
\text { Delta trap }\end{array}$ & $\begin{array}{c}1.25 \\
(1.49)^{\mathrm{c}}\end{array}$ & $\begin{array}{c}1.00 \\
(1.41)^{\mathrm{c}}\end{array}$ & $\begin{array}{c}1.00 \\
(1.41)^{\mathrm{b}}\end{array}$ & $\begin{array}{c}1.75 \\
(1.65)^{\mathrm{c}}\end{array}$ & $\begin{array}{c}1.00 \\
(1.41)^{\mathrm{c}}\end{array}$ & $\begin{array}{c}1.50 \\
(1.57)^{\mathrm{b}}\end{array}$ & $\begin{array}{c}0.00 \\
(1.00)^{\mathrm{c}}\end{array}$ & $\begin{array}{c}0.50 \\
(1.21)^{\mathrm{b}}\end{array}$ & $\begin{array}{c}1.25 \\
(1.49)^{\mathrm{b}}\end{array}$ & $\begin{array}{c}0.50 \\
(1.21)^{\mathrm{b}}\end{array}$ & $\begin{array}{c}0.50 \\
(1.21)^{\mathrm{b}}\end{array}$ & $\begin{array}{c}1.00 \\
(1.39)^{\mathrm{b}}\end{array}$ & $\begin{array}{c}1.00 \\
(1.41)^{\mathrm{b}}\end{array}$ & $\begin{array}{c}0.75 \\
(1.31)^{\mathrm{b}}\end{array}$ & $\begin{array}{c}0.75 \\
(1.31)^{\mathrm{c}}\end{array}$ \\
\hline $\begin{array}{c}\mathrm{T} 4 \\
\text { Bottle trap }\end{array}$ & $\begin{array}{c}2.15 \\
(1.93)^{\mathrm{ab}}\end{array}$ & $\begin{array}{c}1.50 \\
(1.57)^{\mathrm{c}}\end{array}$ & $\begin{array}{c}1.25 \\
(1.49)^{\mathrm{ab}}\end{array}$ & $\begin{array}{c}2.25 \\
(1.80)^{\mathrm{c}}\end{array}$ & $\begin{array}{c}2.00 \\
(1.73)^{\mathrm{b}}\end{array}$ & $\begin{array}{c}1.75 \\
(1.65)^{\mathrm{b}}\end{array}$ & $\begin{array}{c}1.25 \\
(1.49)^{\mathrm{ab}}\end{array}$ & $\begin{array}{c}0.75 \\
(1.31)^{\mathrm{b}}\end{array}$ & $\begin{array}{c}1.25 \\
(1.49)^{\mathrm{b}}\end{array}$ & $\begin{array}{c}0.25 \\
(1.10)^{\mathrm{b}}\end{array}$ & $\begin{array}{c}1.00 \\
(1.41)^{\mathrm{b}}\end{array}$ & $\begin{array}{c}1.50 \\
(1.57)^{\mathrm{ab}}\end{array}$ & $\begin{array}{c}1.00 \\
(1.41)^{\mathrm{b}}\end{array}$ & $\begin{array}{c}1.50 \\
(1.57)^{\mathrm{ab}}\end{array}$ & $\begin{array}{c}1.25 \\
(1.49)^{\mathrm{bc}}\end{array}$ \\
\hline $\begin{array}{c}\text { T5 } \\
\text { Can trap }\end{array}$ & $\begin{array}{c}5.50 \\
(2.54)^{\mathrm{a}}\end{array}$ & $\begin{array}{c}4.00 \\
(2.22)^{\mathrm{b}}\end{array}$ & $\begin{array}{c}3.75 \\
(2.18)^{\mathrm{a}}\end{array}$ & $\begin{array}{c}3.75 \\
(2.17)^{\mathrm{b}}\end{array}$ & $\begin{array}{c}2.25 \\
(1.79)^{\mathrm{b}}\end{array}$ & $\begin{array}{c}2.25 \\
(1.80)^{\mathrm{b}}\end{array}$ & $\begin{array}{c}0.50 \\
(1.21)^{\mathrm{c}}\end{array}$ & $\begin{array}{c}1.00 \\
(1.39)^{\mathrm{b}}\end{array}$ & $\begin{array}{c}2.00 \\
(1.72)^{\mathrm{b}}\end{array}$ & $\begin{array}{c}1.50 \\
(1.57)^{\mathrm{ab}}\end{array}$ & $\begin{array}{c}1.25 \\
(1.49)^{\mathrm{b}}\end{array}$ & $\begin{array}{c}1.75 \\
(1.65)^{\mathrm{ab}}\end{array}$ & $\begin{array}{c}1.25 \\
(1.49)^{\mathrm{ab}}\end{array}$ & $\begin{array}{c}2.00 \\
(1.72)^{\mathrm{a}}\end{array}$ & $\begin{array}{c}2.25 \\
(1.79)^{\mathrm{ab}}\end{array}$ \\
\hline CD@ $@ 5 \%$ & 0.22 & 0.22 & 0.12 & 0.20 & 0.16 & 0.14 & 0.23 & 0.26 & 0.23 & 0.35 & 0.19 & 0.19 & 0.15 & 0.21 & 0.24 \\
\hline SE.m \pm & 0.04 & 0.04 & $\mathbf{0 . 0 3}$ & 0.04 & 0.02 & 0.04 & 0.07 & 0.06 & 0.02 & 0.08 & 0.04 & 0.02 & 0.03 & 0.06 & 0.06 \\
\hline
\end{tabular}

DAIT- Days After Installation of Traps; Figures in parentheses $\sqrt{x}+1$ transformed values

Continued... 
Continued..

\begin{tabular}{|c|c|c|c|c|c|c|c|c|c|c|c|c|c|c|c|c|}
\hline \multirow[b]{2}{*}{ Treatment } & \multicolumn{16}{|c|}{ Number of moths caught/trap (in five days) } \\
\hline & $\begin{array}{c}80 \\
\text { DAIT }\end{array}$ & $\begin{array}{c}85 \\
\text { DAIT }\end{array}$ & $\begin{array}{c}90 \\
\text { DAIT }\end{array}$ & $\begin{array}{c}95 \\
\text { DAIT }\end{array}$ & $\begin{array}{c}100 \\
\text { DAIT }\end{array}$ & $\begin{array}{c}105 \\
\text { DAIT }\end{array}$ & $\begin{array}{c}110 \\
\text { DAIT }\end{array}$ & $\begin{array}{c}115 \\
\text { DAIT }\end{array}$ & $\begin{array}{c}120 \\
\text { DAIT }\end{array}$ & $\begin{array}{c}125 \\
\text { DAIT }\end{array}$ & $\begin{array}{c}130 \\
\text { DAIT }\end{array}$ & $\begin{array}{c}135 \\
\text { DAIT }\end{array}$ & $\begin{array}{c}140 \\
\text { DAIT }\end{array}$ & $\begin{array}{c}145 \\
\text { DAIT }\end{array}$ & $\begin{array}{c}150 \\
\text { DAIT }\end{array}$ & Mean \\
\hline $\begin{array}{c}\text { T1 } \\
\text { Water trap }\end{array}$ & $\begin{array}{c}2.00 \\
(1.73)^{\mathrm{a}}\end{array}$ & $\begin{array}{c}5.00 \\
(2.45)^{\mathrm{a}}\end{array}$ & $\begin{array}{c}3.00 \\
(2.00)^{\mathrm{a}}\end{array}$ & $\begin{array}{c}3.00 \\
(2.00)^{\mathrm{a}}\end{array}$ & $\begin{array}{c}4.75 \\
(2.40)^{\mathrm{a}}\end{array}$ & $\begin{array}{c}0.75 \\
(1.31)^{\mathrm{a}}\end{array}$ & $\begin{array}{c}0.00 \\
(1.31)^{\mathrm{a}}\end{array}$ & $\begin{array}{c}0.00 \\
(1.31)^{\mathrm{a}}\end{array}$ & $\begin{array}{c}1.50 \\
(1.54)^{\mathrm{a}}\end{array}$ & $\begin{array}{c}2.75 \\
(1.93)^{\mathrm{a}}\end{array}$ & $\begin{array}{c}6.00 \\
(2.64)^{\mathrm{a}}\end{array}$ & $\begin{array}{c}2.50 \\
(2.50)^{\mathrm{a}}\end{array}$ & $\begin{array}{c}2.00 \\
(2.50)^{\mathrm{a}}\end{array}$ & $\begin{array}{c}2.50 \\
(1.82)^{\mathrm{a}}\end{array}$ & $\begin{array}{c}2.50 \\
(1.82)^{\mathrm{a}}\end{array}$ & $\begin{array}{c}3.08 \\
(2.03)^{\mathrm{a}}\end{array}$ \\
\hline $\begin{array}{c}\mathrm{T2} \\
\text { Sleeve trap }\end{array}$ & $\begin{array}{c}1.00 \\
(1.41)^{\mathrm{c}}\end{array}$ & $\begin{array}{c}1.50 \\
(1.57)^{\mathrm{b}}\end{array}$ & $\begin{array}{c}0.75 \\
(1.31)^{\mathrm{b}}\end{array}$ & $\begin{array}{c}1.00 \\
(1.41)^{\mathrm{c}}\end{array}$ & $\begin{array}{c}1.00 \\
(1.41)^{\mathrm{cd}}\end{array}$ & $\begin{array}{c}0.25 \\
(1.10)^{\mathrm{a}}\end{array}$ & $\begin{array}{c}0.00 \\
(1.10)^{\mathrm{a}}\end{array}$ & $\begin{array}{c}0.00 \\
(1.10)^{\mathrm{a}}\end{array}$ & $\begin{array}{c}0.25 \\
(1.10)^{\mathrm{a}}\end{array}$ & $\begin{array}{c}0.75 \\
(1.29)^{\mathrm{b}}\end{array}$ & $\begin{array}{c}0.75 \\
(1.31)^{\mathrm{cd}}\end{array}$ & $\begin{array}{c}0.50 \\
(0.50)^{\mathrm{a}}\end{array}$ & $\begin{array}{c}0.75 \\
(0.50)^{\mathrm{a}}\end{array}$ & $\begin{array}{c}0.50 \\
(1.18)^{\mathrm{a}}\end{array}$ & $\begin{array}{c}0.50 \\
(1.18)^{\mathrm{a}}\end{array}$ & $\begin{array}{c}0.96 \\
(1.33)^{\mathrm{c}}\end{array}$ \\
\hline $\begin{array}{c}\mathrm{T} 3 \\
\text { Delta trap }\end{array}$ & $\begin{array}{c}1.00 \\
(1.41)^{\mathrm{c}}\end{array}$ & $\begin{array}{c}0.50 \\
(1.22)^{\mathrm{c}}\end{array}$ & $\begin{array}{c}0.75 \\
(1.31)^{\mathrm{b}}\end{array}$ & $\begin{array}{c}0.75 \\
(1.31)^{\mathrm{c}}\end{array}$ & $\begin{array}{c}0.50 \\
(1.21)^{\mathrm{d}}\end{array}$ & $\begin{array}{c}0.25 \\
(1.10)^{\mathrm{a}}\end{array}$ & $\begin{array}{c}0.00 \\
(1.10)^{\mathrm{a}}\end{array}$ & $\begin{array}{c}0.00 \\
(1.10)^{\mathrm{a}}\end{array}$ & $\begin{array}{c}0.00 \\
(1.00)^{\mathrm{a}}\end{array}$ & $\begin{array}{c}0.50 \\
(1.21)^{\mathrm{b}}\end{array}$ & $\begin{array}{c}0.50 \\
(1.21)^{\mathrm{d}}\end{array}$ & $\begin{array}{c}0.50 \\
(0.50)^{\mathrm{a}}\end{array}$ & $\begin{array}{c}1.50 \\
(0.50)^{\mathrm{a}}\end{array}$ & $\begin{array}{c}0.50 \\
(1.21)^{\mathrm{a}}\end{array}$ & $\begin{array}{c}0.50 \\
(1.21)^{\mathrm{a}}\end{array}$ & $\begin{array}{c}0.72 \\
(1.24)^{\mathrm{c}}\end{array}$ \\
\hline $\begin{array}{c}\text { T4 } \\
\text { Bottle trap }\end{array}$ & $\begin{array}{c}1.00 \\
(1.41)^{\mathrm{c}}\end{array}$ & $\begin{array}{c}1.00 \\
(1.41)^{\mathrm{bc}}\end{array}$ & $\begin{array}{c}1.25 \\
(1.49)^{\mathrm{b}}\end{array}$ & $\begin{array}{c}0.75 \\
(1.31)^{\mathrm{c}}\end{array}$ & $\begin{array}{c}1.25 \\
(1.49)^{\mathrm{c}}\end{array}$ & $\begin{array}{c}0.00 \\
(1.00)^{\mathrm{a}}\end{array}$ & $\begin{array}{c}0.00 \\
(1.00)^{\mathrm{a}}\end{array}$ & $\begin{array}{c}0.00 \\
(1.00)^{\mathrm{a}}\end{array}$ & $\begin{array}{c}0.00 \\
(1.00)^{\mathrm{a}}\end{array}$ & $\begin{array}{c}0.75 \\
(1.31)^{\mathrm{b}}\end{array}$ & $\begin{array}{c}1.50 \\
(1.57)^{\mathrm{c}}\end{array}$ & $\begin{array}{c}0.50 \\
(0.50)^{\mathrm{a}}\end{array}$ & $\begin{array}{c}1.50 \\
(0.50)^{\mathrm{a}}\end{array}$ & $\begin{array}{c}0.50 \\
(1.21)^{\mathrm{a}}\end{array}$ & $\begin{array}{c}0.50 \\
(1.21)^{\mathrm{a}}\end{array}$ & $\begin{array}{c}1.06 \\
(1.35)^{\mathrm{ab}}\end{array}$ \\
\hline $\begin{array}{c}\text { T5 } \\
\text { Can trap }\end{array}$ & $\begin{array}{c}1.50 \\
(1.57)^{\mathrm{b}}\end{array}$ & $\begin{array}{c}4.50 \\
(2.34)^{\mathrm{a}}\end{array}$ & $\begin{array}{c}2.75 \\
(1.93)^{\mathrm{a}}\end{array}$ & $\begin{array}{c}2.25 \\
(1.80)^{\mathrm{b}}\end{array}$ & $\begin{array}{c}2.00 \\
(1.73)^{\mathrm{b}}\end{array}$ & $\begin{array}{c}0.75 \\
(1.31)^{\mathrm{a}}\end{array}$ & $\begin{array}{c}0.00 \\
(1.31)^{\mathrm{a}}\end{array}$ & $\begin{array}{c}0.00 \\
(1.31)^{\mathrm{a}}\end{array}$ & $\begin{array}{c}1.25 \\
(1.36)^{\mathrm{a}}\end{array}$ & $\begin{array}{c}1.25 \\
(1.49)^{\mathrm{b}}\end{array}$ & $\begin{array}{c}2.50 \\
(1.87)^{b}\end{array}$ & $\begin{array}{c}1.75 \\
(1.75)^{\mathrm{a}}\end{array}$ & $\begin{array}{c}1.75 \\
(1.75)^{\mathrm{a}}\end{array}$ & $\begin{array}{c}1.75 \\
(1.64)^{\mathrm{a}}\end{array}$ & $\begin{array}{c}1.75 \\
(1.64)^{\mathrm{a}}\end{array}$ & $\begin{array}{c}2.03 \\
(1.72)^{\mathrm{ab}}\end{array}$ \\
\hline CD@ 5\% & 0.09 & 0.17 & 0.18 & 0.17 & 0.15 & NS & NS & NS & NS & 0.24 & 0.23 & NS & NS & NS & NS & 0.29 \\
\hline SE.m \pm & 0.02 & 0.05 & 0.05 & 0.04 & 0.03 & 0.11 & 0.12 & 0.12 & 0.80 & 0.06 & 0.06 & 0.68 & 0.66 & 0.67 & 0.67 & 0.06 \\
\hline
\end{tabular}

DAIT- Days After Installation of Traps; Figures in parentheses $\sqrt{\mathrm{x}+1}$ transformed values 
Table.2 Influence of moth trapping through different trap models on shoot damage (\%) due to L. orbonalis monitoring in brinjal crop

\begin{tabular}{|c|c|c|c|c|c|c|c|c|c|c|c|c|c|c|c|}
\hline \multirow[b]{2}{*}{ Treatment } & \multicolumn{15}{|c|}{ Shoot damage (\%) as influenced by moth trapping. } \\
\hline & $\begin{array}{c}5 \\
\text { DAIT }\end{array}$ & $\begin{array}{c}10 \\
\text { DAIT }\end{array}$ & $\begin{array}{c}15 \\
\text { DAIT }\end{array}$ & $\begin{array}{c}20 \\
\text { DAIT }\end{array}$ & $\begin{array}{c}25 \\
\text { DAIT }\end{array}$ & $\begin{array}{c}30 \\
\text { DAIT }\end{array}$ & $\begin{array}{c}35 \\
\text { DAIT }\end{array}$ & $\begin{array}{c}40 \\
\text { DAIT }\end{array}$ & $\begin{array}{c}45 \\
\text { DAIT }\end{array}$ & $\begin{array}{c}50 \\
\text { DAIT }\end{array}$ & $\begin{array}{c}55 \\
\text { DAIT }\end{array}$ & $\begin{array}{c}60 \\
\text { DAIT }\end{array}$ & $\begin{array}{c}65 \\
\text { DAIT }\end{array}$ & $\begin{array}{c}70 \\
\text { DAIT }\end{array}$ & $\begin{array}{c}75 \\
\text { DAIT }\end{array}$ \\
\hline $\begin{array}{c}\mathrm{T} 1 \\
\text { Water trap }\end{array}$ & $\begin{array}{c}1.38 \\
(6.71)^{\mathrm{a}}\end{array}$ & $\begin{array}{l}2.75 \\
(9.51)^{\mathrm{a}}\end{array}$ & $\begin{array}{c}4.39 \\
12.02^{\mathrm{a}}\end{array}$ & $\begin{array}{c}5.54 \\
(13.61)^{\mathrm{a}}\end{array}$ & $\begin{array}{c}6.94 \\
(6.71)^{\mathrm{a}}\end{array}$ & $\begin{array}{c}7.62 \\
15.98)^{\mathrm{a}}\end{array}$ & $\begin{array}{c}7.36 \\
(15.69)^{\mathrm{a}}\end{array}$ & $\begin{array}{c}7.15 \\
(23.32)^{\mathrm{a}}\end{array}$ & $\begin{array}{c}9.33 \\
(17.77)^{\mathrm{a}}\end{array}$ & $\begin{array}{c}8.35 \\
(16.61)^{\mathrm{a}}\end{array}$ & $\begin{array}{c}8.90 \\
(17.28)^{\mathrm{a}}\end{array}$ & $\begin{array}{c}7.76 \\
(16.06)^{\mathrm{a}}\end{array}$ & $\begin{array}{c}6.80 \\
(14.97)^{\mathrm{a}}\end{array}$ & $\begin{array}{c}7.76 \\
(16.06)^{a}\end{array}$ & $\begin{array}{c}3.06 \\
(10.05)^{\mathrm{a}}\end{array}$ \\
\hline $\begin{array}{c}\mathrm{T} 2 \\
\text { Sleeve trap }\end{array}$ & $\begin{array}{c}5.14 \\
(13.08)^{b}\end{array}$ & $\begin{array}{c}8.22 \\
(16.62)^{b}\end{array}$ & $\begin{array}{c}6.89 \\
(15.19)^{\text {bc }}\end{array}$ & $\begin{array}{c}7.26 \\
(15.55)^{\mathrm{ab}}\end{array}$ & $\begin{array}{c}9.53 \\
(14.24)^{\mathrm{a}}\end{array}$ & $\begin{array}{c}9.65 \\
(18.06)^{\mathrm{ab}}\end{array}$ & $\begin{array}{c}9.71 \\
(18.14)^{b c}\end{array}$ & $\begin{array}{c}9.65 \\
(25.97)^{b c}\end{array}$ & $\begin{array}{c}10.58 \\
(18.97)^{\mathrm{a}}\end{array}$ & $\begin{array}{c}10.15 \\
(18.52)^{\mathrm{a}}\end{array}$ & $\begin{array}{c}9.73 \\
(18.13)^{\mathrm{ab}}\end{array}$ & $\begin{array}{c}9.01 \\
(17.42)^{\mathrm{a}}\end{array}$ & $\begin{array}{c}9.61 \\
(18.01)^{\mathrm{ab}}\end{array}$ & $\begin{array}{c}9.86 \\
(18.29) \mathrm{ab}\end{array}$ & $\begin{array}{c}9.33 \\
(17.77)^{\mathrm{b}}\end{array}$ \\
\hline $\begin{array}{c}\mathrm{T} 3 \\
\text { Delta trap }\end{array}$ & $\begin{array}{c}7.47 \\
(14.24)^{\mathrm{c}}\end{array}$ & $\begin{array}{c}11.57 \\
(19.88)^{\mathrm{c}}\end{array}$ & $\begin{array}{c}7.80 \\
(16.19)^{\mathrm{c}}\end{array}$ & $\begin{array}{c}7.96 \\
(16.31)^{b}\end{array}$ & $\begin{array}{c}10.40 \\
(13.08)^{\mathrm{a}}\end{array}$ & $\begin{array}{c}11.63 \\
(19.91)^{\mathrm{b}}\end{array}$ & $\begin{array}{c}10.80 \\
(19.17)^{\mathrm{c}}\end{array}$ & $\begin{array}{c}11.01 \\
(25.20)^{\mathrm{c}}\end{array}$ & $\begin{array}{c}10.70 \\
(19.07)^{\mathrm{a}}\end{array}$ & $\begin{array}{c}10.36 \\
(18.76)^{\mathrm{a}}\end{array}$ & $\begin{array}{c}11.57 \\
(19.88)^{b}\end{array}$ & $\begin{array}{c}11.57 \\
(19.88)^{b}\end{array}$ & $\begin{array}{c}11.60 \\
(19.76)^{b}\end{array}$ & $\begin{array}{c}11.57 \\
(19.88)^{b}\end{array}$ & $\begin{array}{c}9.47 \\
(17.82)^{\mathrm{a}}\end{array}$ \\
\hline $\begin{array}{c}\mathrm{T} 4 \\
\text { Bottle trap }\end{array}$ & $\begin{array}{c}1.97 \\
(7.09)^{\mathrm{a}}\end{array}$ & $\begin{array}{c}7.76 \\
(16.06)^{\mathrm{b}}\end{array}$ & $\begin{array}{c}5.19 \\
(13.08)^{\mathrm{c}}\end{array}$ & $\begin{array}{c}7.08 \\
(15.37)^{\mathrm{ab}}\end{array}$ & $\begin{array}{c}9.18 \\
(17.90)^{\mathrm{a}}\end{array}$ & $\begin{array}{c}9.07 \\
(17.43)^{\mathrm{b}}\end{array}$ & $\begin{array}{c}9.07 \\
(17.43)^{\mathrm{abc}}\end{array}$ & $\begin{array}{c}8.64 \\
(24.64)^{\mathrm{ab}}\end{array}$ & $\begin{array}{c}10.81 \\
(19.16)^{\mathrm{a}}\end{array}$ & $\begin{array}{c}10.49 \\
(18.87)^{\mathrm{a}}\end{array}$ & $\begin{array}{c}9.23 \\
(17.67)^{\mathrm{a}}\end{array}$ & $\begin{array}{c}8.88 \\
(17.32)^{\mathrm{a}}\end{array}$ & $\begin{array}{c}9.13 \\
(17.57)^{\mathrm{ab}}\end{array}$ & $\begin{array}{c}8.85 \\
(17.28)^{\mathrm{a}}\end{array}$ & $\begin{array}{c}8.30 \\
(16.70)^{\mathrm{b}}\end{array}$ \\
\hline $\begin{array}{c}\text { T5 } \\
\text { Can trap }\end{array}$ & $\begin{array}{c}1.72 \\
(6.81)^{\mathrm{a}}\end{array}$ & $\begin{array}{c}4.12 \\
(11.52)^{\mathrm{a}}\end{array}$ & $\begin{array}{c}4.32 \\
(11.76)^{\mathrm{a}}\end{array}$ & $\begin{array}{c}5.60 \\
(13.67)^{\mathrm{a}}\end{array}$ & $\begin{array}{c}7.97 \\
(16.81)^{\mathrm{a}}\end{array}$ & $\begin{array}{c}8.52 \\
(16.97)^{\mathrm{a}}\end{array}$ & $\begin{array}{c}8.19 \\
(16.63)^{\mathrm{ab}}\end{array}$ & $\begin{array}{c}8.31 \\
(24.06)^{\mathrm{ab}}\end{array}$ & $\begin{array}{c}10.11 \\
(18.51)^{\mathrm{a}}\end{array}$ & $\begin{array}{c}9.40 \\
(17.79)^{\mathrm{a}}\end{array}$ & $\begin{array}{c}8.22 \\
(16.62)^{\mathrm{a}}\end{array}$ & $\begin{array}{c}8.22 \\
(16.62)^{\mathrm{a}}\end{array}$ & $\begin{array}{c}7.60 \\
(15.91)^{\mathrm{a}}\end{array}$ & $\begin{array}{c}8.22 \\
(16.62)^{\mathrm{a}}\end{array}$ & $\begin{array}{c}8.01 \\
(16.36)^{b}\end{array}$ \\
\hline $\begin{array}{c}\mathrm{T} 6 \\
\text { Control }\end{array}$ & $\begin{array}{c}7.26 \\
(15.55)^{\mathrm{c}}\end{array}$ & $\begin{array}{c}9.65 \\
(18.06)^{\mathrm{c}}\end{array}$ & $\begin{array}{c}7.36 \\
(15.69)^{\mathrm{c}}\end{array}$ & $\begin{array}{c}7.76 \\
(16.06)^{b}\end{array}$ & $\begin{array}{c}10.49 \\
(18.87)^{\mathrm{a}}\end{array}$ & $\begin{array}{c}11.57 \\
(19.88)^{\mathrm{b}}\end{array}$ & $\begin{array}{c}10.70 \\
(19.07)^{\mathrm{c}}\end{array}$ & $\begin{array}{c}10.36 \\
(18.76)^{\mathrm{c}}\end{array}$ & $\begin{array}{c}10.80 \\
(19.17)^{\mathrm{a}}\end{array}$ & $\begin{array}{c}10.40 \\
(19.08)^{\mathrm{a}}\end{array}$ & $\begin{array}{c}11.01 \\
(25.20)^{\mathrm{b}}\end{array}$ & $\begin{array}{c}11.60 \\
(19.76)^{b}\end{array}$ & $\begin{array}{c}11.63 \\
(19.91)^{b}\end{array}$ & $\begin{array}{c}11.01 \\
(25.20)^{\mathrm{b}}\end{array}$ & $\begin{array}{c}9.73 \\
(18.13)^{b}\end{array}$ \\
\hline CD@ $9 \%$ & 2.12 & 2.03 & 2.04 & 1.46 & NS & 1.55 & 1.38 & 1.07 & NS & NS & 1.46 & 1.56 & 2.14 & 1.52 & 1.89 \\
\hline SE.m \pm & 0.45 & 0.48 & 0.37 & 0.32 & 2.13 & 0.37 & 0.34 & 0.39 & 0.09 & 0.15 & 0.46 & 0.28 & 0.41 & 0.39 & 0.44 \\
\hline
\end{tabular}

DAIT- Days After Installation of Traps; Figures in parentheses are arc sine transformed values.

Contdinued... 


\begin{tabular}{|c|c|c|c|c|c|c|c|c|c|c|c|c|c|c|c|c|}
\hline \multirow[b]{2}{*}{ Treatment } & \multicolumn{16}{|c|}{ Shoot damage (\%) as influenced by moth trapping. } \\
\hline & $\begin{array}{c}80 \\
\text { DAIT }\end{array}$ & $\begin{array}{c}85 \\
\text { DAIT }\end{array}$ & $\begin{array}{c}90 \\
\text { DAIT }\end{array}$ & $\begin{array}{c}95 \\
\text { DAIT }\end{array}$ & $\begin{array}{c}100 \\
\text { DAIT }\end{array}$ & $\begin{array}{c}105 \\
\text { DAIT }\end{array}$ & $\begin{array}{c}110 \\
\text { DAIT }\end{array}$ & $\begin{array}{c}115 \\
\text { DAIT }\end{array}$ & $\begin{array}{c}120 \\
\text { DAIT }\end{array}$ & $\begin{array}{c}125 \\
\text { DAIT }\end{array}$ & $\begin{array}{c}130 \\
\text { DAIT }\end{array}$ & $\begin{array}{c}135 \\
\text { DAIT }\end{array}$ & $\begin{array}{c}140 \\
\text { DAIT }\end{array}$ & $\begin{array}{c}145 \\
\text { DAIT }\end{array}$ & $\begin{array}{c}150 \\
\text { DAIT }\end{array}$ & Mean \\
\hline $\begin{array}{c}\mathrm{T} 1 \\
\text { Water trap }\end{array}$ & $\begin{array}{c}7.51 \\
(15.74)^{\mathrm{a}}\end{array}$ & $\begin{array}{c}0.09 \\
(0.87)^{\mathrm{a}}\end{array}$ & $\begin{array}{c}1.62 \\
(7.11)^{\mathrm{a}}\end{array}$ & $\begin{array}{c}5.35 \\
(13.35)^{\mathrm{a}}\end{array}$ & $\begin{array}{c}5.03 \\
(12.94)^{\mathrm{a}}\end{array}$ & $\begin{array}{c}3.76 \\
(11.16)^{\mathrm{a}}\end{array}$ & $\begin{array}{c}3.26 \\
(10.35)^{\mathrm{a}}\end{array}$ & $\begin{array}{c}3.61 \\
(10.88)^{\mathrm{a}}\end{array}$ & $\begin{array}{c}4.41 \\
(12.08)^{\mathrm{a}}\end{array}$ & $\begin{array}{c}5.43 \\
(13.45)^{\mathrm{a}}\end{array}$ & $\begin{array}{c}5.58 \\
(13.59)^{\mathrm{a}}\end{array}$ & $\begin{array}{c}6.51 \\
(14.78)^{\mathrm{a}}\end{array}$ & $\begin{array}{c}6.94 \\
(15.21)^{\mathrm{a}}\end{array}$ & $\begin{array}{c}7.51 \\
(15.82)^{\mathrm{a}}\end{array}$ & $\begin{array}{c}2.81 \\
(9.51)^{\mathrm{a}}\end{array}$ & $\begin{array}{c}5.48 \\
(12.97)^{\mathrm{a}}\end{array}$ \\
\hline $\begin{array}{c}\mathrm{T} 2 \\
\text { Sleeve } \\
\text { trap }\end{array}$ & $\begin{array}{c}9.82 \\
(18.22)^{\mathrm{ab}}\end{array}$ & $\begin{array}{c}0.59 \\
(3.09)^{\mathrm{a}}\end{array}$ & $\begin{array}{c}6.40 \\
(14.63)^{\mathrm{b}}\end{array}$ & $\begin{array}{c}9.92 \\
(18.32)^{\mathrm{b}}\end{array}$ & $\begin{array}{c}7.95 \\
(16.36)^{b}\end{array}$ & $\begin{array}{c}8.22 \\
(16.62)_{b}\end{array}$ & $\begin{array}{c}4.13 \\
(11.67)^{\mathrm{ab}}\end{array}$ & $\begin{array}{c}8.09 \\
(16.50)^{\mathrm{b}}\end{array}$ & $\begin{array}{c}6.47 \\
(14.73)^{\mathrm{b}}\end{array}$ & $\begin{array}{c}6.86 \\
(15.15)^{\mathrm{ab}}\end{array}$ & $\begin{array}{c}7.54 \\
(15.90)^{\mathrm{bc}}\end{array}$ & $\begin{array}{c}7.97 \\
(16.36)^{\mathrm{ab}}\end{array}$ & $\begin{array}{c}9.24 \\
(17.69)^{\mathrm{b}}\end{array}$ & $\begin{array}{c}9.33 \\
(17.77)^{\mathrm{ab}}\end{array}$ & $\begin{array}{c}9.33 \\
(17.77)^{b}\end{array}$ & $\begin{array}{c}8.24 \\
(15.44)^{b}\end{array}$ \\
\hline $\begin{array}{c}\mathrm{T} 3 \\
\text { Delta trap }\end{array}$ & $\begin{array}{c}11.07 \\
(19.42)^{\mathrm{a}}\end{array}$ & $\begin{array}{c}0.37 \\
(2.38)^{\mathrm{a}}\end{array}$ & $\begin{array}{c}6.42 \\
(14.67)^{\mathrm{b}}\end{array}$ & $\begin{array}{c}9.33 \\
(17.77)^{\mathrm{b}}\end{array}$ & $\begin{array}{c}8.13 \\
(16.53)^{\mathrm{b}}\end{array}$ & $\begin{array}{c}8.39 \\
(16.80)_{b}\end{array}$ & $\begin{array}{c}6.68 \\
(14.96)^{b}\end{array}$ & $\begin{array}{c}8.47 \\
(16.89)^{\mathrm{a}}\end{array}$ & $\begin{array}{c}6.68 \\
(14.97)^{\mathrm{b}}\end{array}$ & $\begin{array}{c}7.71 \\
(16.09)^{\mathrm{b}}\end{array}$ & $\begin{array}{c}7.90 \\
(16.30)^{\mathrm{c}}\end{array}$ & $\begin{array}{c}9.33 \\
(17.76)^{b}\end{array}$ & $\begin{array}{c}9.25 \\
(17.70)^{\mathrm{b}}\end{array}$ & $\begin{array}{c}11.32 \\
(19.64)^{\mathrm{b}}\end{array}$ & $\begin{array}{c}11.57 \\
(19.88)^{\mathrm{c}}\end{array}$ & $\begin{array}{c}9.16 \\
(17.38)^{b}\end{array}$ \\
\hline $\begin{array}{c}\mathrm{T} 4 \\
\text { Bottle trap }\end{array}$ & $\begin{array}{c}8.91 \\
(17.36)^{\mathrm{ab}}\end{array}$ & $\begin{array}{c}0.30 \\
(2.19)^{\mathrm{a}}\end{array}$ & $\begin{array}{c}1.76 \\
(7.08)^{\mathrm{b}}\end{array}$ & $\begin{array}{c}8.22 \\
(16.62)^{\mathrm{b}}\end{array}$ & $\begin{array}{c}7.07 \\
(15.33)^{\mathrm{b}}\end{array}$ & $\begin{array}{c}7.48 \\
(15.79)_{b}\end{array}$ & $\begin{array}{c}3.85 \\
(11.26)^{\mathrm{a}}\end{array}$ & $\begin{array}{c}4.13 \\
(11.68)^{\mathrm{ab}}\end{array}$ & $\begin{array}{c}5.57 \\
(13.64)^{\mathrm{ab}}\end{array}$ & $\begin{array}{c}5.82 \\
(13.96)^{\mathrm{ab}}\end{array}$ & $\begin{array}{c}6.46 \\
(14.71)^{\mathrm{abc}}\end{array}$ & $\begin{array}{c}7.26 \\
(15.56)^{\mathrm{a}}\end{array}$ & $\begin{array}{c}9.04 \\
(17.49)^{\mathrm{b}}\end{array}$ & $\begin{array}{c}8.99 \\
(17.40)^{\mathrm{a}}\end{array}$ & $\begin{array}{c}8.22 \\
(16.62)^{\mathrm{b}}\end{array}$ & $\begin{array}{c}7.22 \\
(15.01)^{\mathrm{ab}}\end{array}$ \\
\hline $\begin{array}{c}\mathrm{T} 5 \\
\text { Can trap }\end{array}$ & $\begin{array}{c}8.22 \\
(16.62)^{\mathrm{a}}\end{array}$ & $\begin{array}{c}0.19 \\
(1.26)^{\mathrm{a}}\end{array}$ & $\begin{array}{c}7.46 \\
(15.82)^{\mathrm{b}}\end{array}$ & $\begin{array}{c}7.76 \\
(16.06)^{\mathrm{b}}\end{array}$ & $\begin{array}{c}5.11 \\
(13.06)^{\mathrm{a}}\end{array}$ & $\begin{array}{c}4.23 \\
(11.84)_{\mathrm{a}}\end{array}$ & $\begin{array}{c}3.46 \\
(10.68)^{b}\end{array}$ & $\begin{array}{c}4.13 \\
(11.68)^{\mathrm{ab}}\end{array}$ & $\begin{array}{c}5.21 \\
(13.14)^{\mathrm{a}}\end{array}$ & $\begin{array}{c}5.61 \\
(13.63)^{\mathrm{a}}\end{array}$ & $\begin{array}{c}5.87 \\
(13.97)^{\mathrm{ab}}\end{array}$ & $\begin{array}{c}6.80 \\
(15.08)^{\mathrm{b}}\end{array}$ & $\begin{array}{c}8.13 \\
(16.53)^{\mathrm{ab}}\end{array}$ & $\begin{array}{c}8.22 \\
(16.62)^{\mathrm{a}}\end{array}$ & $\begin{array}{c}7.76 \\
(16.06)^{\mathrm{b}}\end{array}$ & $\begin{array}{c}6.56 \\
(14.29)^{\mathrm{ab}}\end{array}$ \\
\hline $\begin{array}{c}\text { T6 } \\
\text { Control }\end{array}$ & $\begin{array}{c}11.63 \\
(19.91)^{\mathrm{b}}\end{array}$ & $\begin{array}{c}0.69 \\
(3.19)^{\mathrm{a}}\end{array}$ & $\begin{array}{c}6.47 \\
(14.73)^{\mathrm{b}}\end{array}$ & $\begin{array}{c}9.53 \\
(14.24)^{\mathrm{b}}\end{array}$ & $\begin{array}{c}8.22 \\
(16.62)^{b}\end{array}$ & $\begin{array}{c}8.39 \\
(16.80)_{b}\end{array}$ & $\begin{array}{c}5.60 \\
(13.67)^{\mathrm{b}}\end{array}$ & $\begin{array}{c}9.04 \\
(17.49)^{b}\end{array}$ & $\begin{array}{c}6.42 \\
(14.67)^{\mathrm{b}}\end{array}$ & $\begin{array}{c}7.97 \\
(16.36)^{b}\end{array}$ & $\begin{array}{c}7.95 \\
(16.36)^{\mathrm{c}}\end{array}$ & $\begin{array}{c}8.91 \\
(17.36)^{b}\end{array}$ & $\begin{array}{c}9.92 \\
(18.32)^{\mathrm{b}}\end{array}$ & $\begin{array}{c}11.07 \\
(19.42)^{\mathrm{ab}}\end{array}$ & $\begin{array}{c}11.60 \\
(19.76)^{\mathrm{c}}\end{array}$ & $\begin{array}{c}9.24 \\
(16.32)^{b}\end{array}$ \\
\hline CD@ 5\% & 1.78 & 3.08 & 1.77 & 1.85 & 1.49 & 1.58 & 1.16 & 1.55 & 0.96 & 1.28 & 1.39 & 1.31 & 1.17 & 1.67 & 1.93 & 1.65 \\
\hline SE.m \pm & 0.56 & NS & 0.40 & 0.58 & 0.46 & 0.47 & 0.31 & 0.41 & 0.29 & 0.39 & 0.24 & 0.39 & 0.30 & 0.41 & 0.43 & 0.42 \\
\hline
\end{tabular}

DAIT- Days After Installation of Traps; Figures in parentheses are arc sine transformed values. 


\section{Int.J.Curr.Microbiol.App.Sci (2017) 6(2): 575-585}

Table.3 Influence of moth trapping through different trap models on fruit damage (\%) due to L. orbonalis monitoring in brinjal crop

\begin{tabular}{|c|c|c|c|c|c|c|c|c|c|c|c|c|c|c|c|}
\hline \multirow{3}{*}{ Treatment } & \multicolumn{15}{|c|}{ Fruit damage (\%) as influenced by moth trapping. } \\
\hline & \multicolumn{15}{|c|}{ Pickings } \\
\hline & $\mathbf{1}^{\text {st }}$ & $2^{\text {nd }}$ & $3^{\text {rd }}$ & $4^{\text {th }}$ & $5^{\text {th }}$ & $6^{\text {th }}$ & $7^{\text {th }}$ & $8^{\text {th }}$ & $9^{\text {th }}$ & $10^{\text {th }}$ & $11^{\text {th }}$ & $12^{\text {th }}$ & $13^{\text {th }}$ & $14^{\text {th }}$ & $15^{\text {th }}$ \\
\hline $\mathrm{T} 1$ & 2.81 & 23.32 & 15.95 & 12.42 & 8.13 & 10.21 & 15.40 & 8.58 & 10.65 & 9.73 & 17.35 & 12.46 & 12.53 & 9.83 & 12.21 \\
\hline Water trap & $(9.51)^{\mathrm{a}}$ & $(28.75)^{\mathrm{a}}$ & $(23.38)^{\mathrm{a}}$ & $(20.63)^{\mathrm{a}}$ & $(16.33)^{\mathrm{a}}$ & $(18.18)^{\mathrm{a}}$ & $(23.03)^{\mathrm{a}}$ & $(16.97)^{\mathrm{a}}$ & $(19.04)^{\mathrm{a}}$ & $(18.17)^{\mathrm{a}}$ & $(24.49)^{\mathrm{a}}$ & $(20.36)^{a}$ & $(20.72)^{\mathrm{a}}$ & $(18.26)^{\mathrm{a}}$ & $(20.45)^{\mathrm{a}}$ \\
\hline $\mathrm{T} 2$ & 9.33 & 28.48 & 18.27 & 31.03 & 12.16 & 22.33 & 18.78 & 17.54 & 19.02 & 16.85 & 33.77 & 38.58 & 21.83 & 18.31 & 18.17 \\
\hline Sleeve trap & $(17.77)^{b}$ & $(32.20)^{\mathrm{ab}}$ & $(25.25)^{\mathrm{a}}$ & $(33.69)^{\mathrm{b}}$ & $(20.36)^{\mathrm{bc}}$ & $(28.09)^{\mathrm{b}}$ & $(25.60)^{\mathrm{ab}}$ & $(24.71)^{b}$ & $(25.71)^{\mathrm{bc}}$ & $(24.16)^{b}$ & $(35.51)^{\mathrm{c}}$ & $(38.39)^{\mathrm{c}}$ & $(27.67)^{\mathrm{b}}$ & $(25.16)^{\mathrm{c}}$ & $(25.09)^{\mathrm{ab}}$ \\
\hline $\mathrm{T} 3$ & 11.57 & 32.16 & 30.91 & 32.23 & 14.12 & 21.50 & 19.50 & 19.55 & 23.49 & 17.23 & 35.42 & 39.33 & 23.93 & 20.71 & 21.18 \\
\hline Delta trap & $(19.88)^{\mathrm{c}}$ & $(34.53)^{\mathrm{b}}$ & $(33.65)^{b}$ & $(34.50)^{\mathrm{b}}$ & $(22.03)^{\mathrm{c}}$ & $(26.91)^{\mathrm{ab}}$ & $(26.20)^{\mathrm{b}}$ & $(26.22)^{\mathrm{b}}$ & $(28.96)^{\mathrm{c}}$ & $(24.26)^{\mathrm{b}}$ & $(36.49)^{\mathrm{c}}$ & $(38.80)^{\mathrm{c}}$ & $(29.22)^{\mathrm{b}}$ & $(27.06)^{\mathrm{bc}}$ & $(27.17)^{b}$ \\
\hline $\mathrm{T} 4$ & 8.22 & 26.77 & 17.03 & 23.80 & 11.02 & 13.80 & 19.75 & 16.84 & 15.05 & 13.08 & 28.82 & 27.78 & 22.03 & 15.35 & 18.00 \\
\hline Bottle trap & $(16.62)^{b}$ & $(31.15)^{\mathrm{ab}}$ & $(24.36)^{\mathrm{a}}$ & $(29.19)^{\mathrm{ab}}$ & $(19.38)^{\mathrm{abc}}$ & $(21.64)^{\mathrm{ab}}$ & $(26.38)^{\mathrm{b}}$ & $(24.18)^{\mathrm{b}}$ & $(22.80)^{\mathrm{ab}}$ & $(21.19)^{\mathrm{ab}}$ & $(32.33)^{\mathrm{bc}}$ & $(31.66)^{b}$ & $(27.85)^{\mathrm{b}}$ & $(22.93)^{\mathrm{b}}$ & $(25.07)^{\mathrm{ab}}$ \\
\hline $\mathrm{T} 5$ & 7.76 & 24.18 & 15.99 & 18.70 & 10.34 & 11.74 & 15.27 & 15.58 & 13.40 & 12.73 & 22.22 & 15.22 & 14.50 & 14.73 & 15.25 \\
\hline Can trap & $(16.06)^{b}$ & $(29.39)^{\mathrm{a}}$ & $(23.41)^{\mathrm{a}}$ & $(24.86)^{\mathrm{a}}$ & $(18.67)^{\mathrm{ab}}$ & $(19.96)^{\mathrm{ab}}$ & $(22.95)^{\mathrm{a}}$ & $(22.98)^{b}$ & $(21.36)^{\mathrm{a}}$ & $(20.81)^{\mathrm{ab}}$ & $(28.04)^{\mathrm{ab}}$ & $(22.65)^{\mathrm{a}}$ & $(22.38)^{\mathrm{a}}$ & $(22.51)^{\mathrm{b}}$ & $(22.95)^{\mathrm{ab}}$ \\
\hline T6 & 12.46 & 33.11 & 30.49 & 33.51 & 15.05 & 22.03 & 19.59 & 19.54 & 23.93 & 18.27 & 38.58 & 30.91 & 23.32 & 21.50 & 23.32 \\
\hline Control & $(20.36)^{c}$ & $(35.12)^{\mathrm{b}}$ & $(33.46)^{b}$ & $(35.20)^{\mathrm{b}}$ & $(22.80)^{\mathrm{c}}$ & $(27.85)^{\mathrm{ab}}$ & $(26.27)^{\mathrm{b}}$ & $(26.22)^{\mathrm{b}}$ & $(29.22)^{\mathrm{c}}$ & $(25.25)^{\mathrm{b}}$ & $(38.39)^{\mathrm{c}}$ & $(33.65)^{b}$ & $(28.75)^{\mathrm{b}}$ & $(26.91)^{\mathrm{c}}$ & $(28.75)^{\mathrm{b}}$ \\
\hline CD @ 5\% & 1.93 & 2.77 & 2.41 & 4.58 & 2.56 & 4.41 & 1.79 & 2.99 & 2.79 & 2.80 & 3.35 & 4.50 & 3.11 & 2.85 & 3.18 \\
\hline SE.m \pm & 0.61 & 0.81 & 0.11 & 1.53 & 0.79 & 1.02 & 0.39 & 0.99 & 0.93 & 0.75 & 1.53 & 0.99 & 1.02 & 0.88 & 0.95 \\
\hline
\end{tabular}

Figures in parentheses are arc sine transformed values.

\section{Continuedd...}


Int.J.Curr.Microbiol.App.Sci (2017) 6(2): 575-585

\begin{tabular}{|c|c|c|c|c|c|c|c|c|c|c|c|c|c|c|c|c|}
\hline \multirow{3}{*}{ Treatment } & \multicolumn{16}{|c|}{ Fruit damage (\%) as influenced by moth trapping. } \\
\hline & \multicolumn{16}{|c|}{ Pickings } \\
\hline & $16^{\text {th }}$ & $17^{\text {th }}$ & $18^{\text {th }}$ & $1^{\text {th }}$ & $20^{\text {th }}$ & $21^{\text {st }}$ & $22^{\text {nd }}$ & $\mathbf{2 3}^{\text {rd }}$ & $24^{\text {th }}$ & $25^{\text {th }}$ & $26^{\text {th }}$ & $27^{\text {th }}$ & $28^{\text {th }}$ & $29^{\text {th }}$ & $30^{\text {th }}$ & Mean \\
\hline $\begin{array}{c}\text { T1 } \\
\text { Water trap }\end{array}$ & $\begin{array}{c}17.66 \\
(24.78)^{\mathrm{a}}\end{array}$ & $\begin{array}{c}12.44 \\
(20.64)^{\mathrm{a}}\end{array}$ & $\begin{array}{c}21.46 \\
(27.58)^{\mathrm{a}}\end{array}$ & $\begin{array}{c}20.77 \\
(27.10)^{\mathrm{a}}\end{array}$ & $\begin{array}{c}21.45 \\
(27.39)^{\mathrm{a}}\end{array}$ & $\begin{array}{c}18.98 \\
(25.83)^{\mathrm{a}}\end{array}$ & $\begin{array}{c}20.25 \\
(26.74)^{\mathrm{a}}\end{array}$ & $\begin{array}{c}16.33 \\
(23.72)^{\mathrm{a}}\end{array}$ & $\begin{array}{c}19.37 \\
(26.11)^{\mathrm{a}}\end{array}$ & $\begin{array}{c}22.50 \\
(28.30)^{\mathrm{a}}\end{array}$ & $\begin{array}{c}19.20 \\
(25.95)^{\mathrm{a}}\end{array}$ & $\begin{array}{c}16.50 \\
(23.95)^{\mathrm{a}}\end{array}$ & $\begin{array}{c}10.46 \\
(18.81)^{\mathrm{a}}\end{array}$ & $\begin{array}{c}17.52 \\
(24.60)^{\mathrm{a}}\end{array}$ & $\begin{array}{c}14.36 \\
(22.16)^{\mathrm{a}}\end{array}$ & $\begin{array}{c}15.03 \\
(22.40)^{\mathrm{a}}\end{array}$ \\
\hline $\begin{array}{c}\mathrm{T} 2 \\
\text { Sleeve trap }\end{array}$ & $\begin{array}{c}28.33 \\
(32.02)^{b}\end{array}$ & $\begin{array}{c}26.21 \\
(30.79)^{b}\end{array}$ & $\begin{array}{c}30.49 \\
(33.46)^{b}\end{array}$ & $\begin{array}{c}26.58 \\
(30.90)^{b}\end{array}$ & $\begin{array}{c}33.51 \\
(35.20)^{b c}\end{array}$ & $\begin{array}{c}24.32 \\
(29.51)^{b}\end{array}$ & $\begin{array}{c}23.50 \\
(28.99)^{b c}\end{array}$ & $\begin{array}{c}25.46 \\
(30.10)^{b}\end{array}$ & $\begin{array}{c}25.50 \\
(30.21)^{b}\end{array}$ & $\begin{array}{c}25.25 \\
(30.08)^{\mathrm{ab}}\end{array}$ & $\begin{array}{c}28.75 \\
(32.41)^{b}\end{array}$ & $\begin{array}{c}17.55 \\
(24.70)^{\mathrm{a}}\end{array}$ & $\begin{array}{c}18.99 \\
(25.75)^{b}\end{array}$ & $\begin{array}{c}24.21 \\
(29.45)^{b}\end{array}$ & $\begin{array}{c}17.81 \\
(24.90)^{\mathrm{ab}}\end{array}$ & $\begin{array}{c}23.36 \\
(28.59)^{\mathrm{b}}\end{array}$ \\
\hline $\begin{array}{c}\text { T3 } \\
\text { Delta trap }\end{array}$ & $\begin{array}{c}28.64 \\
(32.28)^{b}\end{array}$ & $\begin{array}{c}27.11 \\
(31.05)^{b}\end{array}$ & $\begin{array}{c}33.11 \\
(35.12)^{b}\end{array}$ & $\begin{array}{c}26.46 \\
(30.94)^{b}\end{array}$ & $\begin{array}{c}40.65 \\
(39.58)^{c}\end{array}$ & $\begin{array}{c}24.05 \\
(29.35)^{\mathrm{b}}\end{array}$ & $\begin{array}{c}24.75 \\
(29.83)^{\mathrm{c}}\end{array}$ & $\begin{array}{c}30.44 \\
(33.42)^{\mathrm{b}}\end{array}$ & $\begin{array}{c}27.91 \\
(31.88)^{b}\end{array}$ & $\begin{array}{c}31.15 \\
(33.88)^{b}\end{array}$ & $\begin{array}{c}28.21 \\
(32.04)^{b}\end{array}$ & $\begin{array}{c}25.25 \\
(30.11)^{b}\end{array}$ & $\begin{array}{c}19.59 \\
(26.27)^{b}\end{array}$ & $\begin{array}{c}24.44 \\
(29.59)^{\mathrm{b}}\end{array}$ & $\begin{array}{c}22.00 \\
(27.95)^{\mathrm{b}}\end{array}$ & $\begin{array}{c}25.89 \\
(30.31)^{b}\end{array}$ \\
\hline $\begin{array}{c}\mathrm{T} 4 \\
\text { Bottle trap }\end{array}$ & $\begin{array}{c}22.38 \\
(28.07)^{\mathrm{ab}}\end{array}$ & $\begin{array}{c}23.35 \\
(28.68)^{b}\end{array}$ & $\begin{array}{c}29.71 \\
(32.98)^{b}\end{array}$ & $\begin{array}{c}22.00 \\
(27.96)^{\mathrm{ab}}\end{array}$ & $\begin{array}{c}25.92 \\
(30.55)^{\mathrm{ab}}\end{array}$ & $\begin{array}{c}23.78 \\
(29.17)^{b}\end{array}$ & $\begin{array}{c}22.00 \\
(27.97)^{\mathrm{ab}}\end{array}$ & $\begin{array}{c}24.77 \\
(29.80)^{\mathrm{b}}\end{array}$ & $\begin{array}{c}20.77 \\
(27.10)^{\mathrm{a}}\end{array}$ & $\begin{array}{c}23.90 \\
(29.20)^{\mathrm{a}}\end{array}$ & $\begin{array}{c}22.44 \\
(28.22)^{\mathrm{ab}}\end{array}$ & $\begin{array}{c}17.25 \\
(24.53)^{\mathrm{a}}\end{array}$ & $\begin{array}{c}15.96 \\
(23.45)^{b}\end{array}$ & $\begin{array}{c}21.85 \\
(27.84)^{\mathrm{ab}}\end{array}$ & $\begin{array}{c}15.79 \\
(23.05)^{b}\end{array}$ & $\begin{array}{c}20.31 \\
(24.89)^{\mathrm{ab}}\end{array}$ \\
\hline $\begin{array}{c}\text { T5 } \\
\text { Can trap }\end{array}$ & $\begin{array}{c}18.89 \\
(25.71)^{\mathrm{a}}\end{array}$ & $\begin{array}{c}19.39 \\
(26.12)^{\mathrm{ab}}\end{array}$ & $\begin{array}{c}28.25 \\
(31.97)^{b}\end{array}$ & $\begin{array}{c}21.43 \\
(27.57)^{\mathrm{ab}}\end{array}$ & $\begin{array}{c}22.97 \\
(28.61)^{\mathrm{a}}\end{array}$ & $\begin{array}{c}23.69 \\
(29.11)^{b}\end{array}$ & $\begin{array}{c}21.75 \\
(27.78)^{\mathrm{ab}}\end{array}$ & $\begin{array}{c}22.26 \\
(28.08)^{\mathrm{ab}}\end{array}$ & $\begin{array}{c}20.09 \\
(26.61)^{\mathrm{a}}\end{array}$ & $\begin{array}{c}22.88 \\
(28.53)^{\mathrm{a}}\end{array}$ & $\begin{array}{c}20.75 \\
(26.78)^{\mathrm{a}}\end{array}$ & $\begin{array}{c}16.91 \\
(24.28)^{\mathrm{a}}\end{array}$ & $\begin{array}{c}14.57 \\
(22.29)^{\mathrm{ab}}\end{array}$ & $\begin{array}{c}18.57 \\
(25.48)^{\mathrm{a}}\end{array}$ & $\begin{array}{c}14.86 \\
(22.56)^{\mathrm{a}}\end{array}$ & $\begin{array}{c}17.83 \\
(24.68)^{\mathrm{ab}}\end{array}$ \\
\hline $\begin{array}{c}\text { T6 } \\
\text { Control }\end{array}$ & $\begin{array}{c}28.21 \\
(32.04)^{b}\end{array}$ & $\begin{array}{c}28.75 \\
(32.41)^{b}\end{array}$ & $\begin{array}{c}33.12 \\
(35.13)^{b}\end{array}$ & $\begin{array}{c}27.91 \\
(31.88)^{b}\end{array}$ & $\begin{array}{c}39.33 \\
(38.80)^{\mathrm{c}}\end{array}$ & $\begin{array}{c}24.77 \\
(29.80)^{b}\end{array}$ & $\begin{array}{c}28.75 \\
(32.41)^{\mathrm{c}}\end{array}$ & $\begin{array}{c}29.73 \\
(32.98)^{b}\end{array}$ & $\begin{array}{c}28.34 \\
(32.02)^{b}\end{array}$ & $\begin{array}{c}30.49 \\
(33.46)^{\mathrm{b}}\end{array}$ & $\begin{array}{c}28.82 \\
(32.33)^{b}\end{array}$ & $\begin{array}{c}27.11 \\
(31.05)^{b}\end{array}$ & $\begin{array}{c}18.89 \\
(25.71)^{\mathrm{b}}\end{array}$ & $\begin{array}{c}24.32 \\
(29.51)^{\mathrm{b}}\end{array}$ & $\begin{array}{c}23.35 \\
(28.68)^{c}\end{array}$ & $\begin{array}{c}26.25 \\
(30.88)^{\mathrm{c}}\end{array}$ \\
\hline CD@5\% & 3.58 & 4.50 & 3.44 & 2.30 & 4.55 & 1.69 & 1.08 & 3.41 & 2.49 & 2.38 & 3.21 & 1.73 & 3.03 & 2.74 & 2.77 & 2.90 \\
\hline SE.m \pm & 0.91 & 1.10 & 1.05 & 0.89 & 1.05 & 0.41 & 0.22 & 1.00 & 0.45 & 0.32 & 0.99 & 0.31 & 1.01 & 0.91 & 0.92 & 0.53 \\
\hline
\end{tabular}

Figures in parentheses are arc sine transformed values. 
Table.4 Overall influence on different monitoring traps on brinjal shoot and frut borer, L. orbonalis on shoot damage, fruit damage and their impact on yield in brinjal crop

\begin{tabular}{|c|c|c|c|}
\hline Treatments & $\begin{array}{c}\text { Shoot } \\
\text { damage } \\
(\%)\end{array}$ & $\begin{array}{c}\text { Fruit } \\
\text { damage } \\
(\%)\end{array}$ & $\begin{array}{l}\text { Yield } \\
\left(\mathbf{q} \text { ha }^{-1}\right)\end{array}$ \\
\hline T1: Water trap & $\begin{array}{c}5.48 \\
(12.97)^{\mathrm{a}}\end{array}$ & $\begin{array}{c}15.03 \\
(22.40)^{\mathrm{a}}\end{array}$ & $489.0^{\mathrm{a}}$ \\
\hline T2: Sleeve trap & $\begin{array}{c}8.24 \\
(15.44)^{\mathrm{b}}\end{array}$ & $\begin{array}{c}23.36 \\
(28.59)^{\mathrm{b}}\end{array}$ & $319.0^{\mathrm{b}}$ \\
\hline T3: Delta trap & $\begin{array}{c}9.16 \\
(17.38)^{\mathrm{b}}\end{array}$ & $\begin{array}{c}25.89 \\
(30.31)^{\mathrm{b}}\end{array}$ & $278.0^{\mathrm{b}}$ \\
\hline T4: Bottle trap & $\begin{array}{c}7.22 \\
(15.01)^{\mathrm{ab}}\end{array}$ & $\begin{array}{c}20.31 \\
(24.89)^{\mathrm{ab}}\end{array}$ & $364.0^{\mathrm{b}}$ \\
\hline T5: Can trap & $\begin{array}{c}6.56 \\
(14.29)^{\mathrm{ab}}\end{array}$ & $\begin{array}{c}17.83 \\
(24.68)^{\mathrm{ab}}\end{array}$ & $416.0^{\mathrm{b}}$ \\
\hline T6: Control & $\begin{array}{c}9.24 \\
(16.32)^{b}\end{array}$ & $\begin{array}{c}26.25 \\
(30.88)^{\mathrm{c}}\end{array}$ & $2710^{\mathrm{b}}$ \\
\hline CD@ $@ 5 \%$ & 1.65 & 2.90 & 702.8 \\
\hline SE.m \pm & 0.42 & 0.53 & 221.29 \\
\hline
\end{tabular}

Results are in conformity with Rahman et al., (2009), who reported that spraying of chemical at two days interval, mechanical control and using pheromone trap performed the best in all respects ensuring the lowest shoot $(6.27 \%)$ and fruit $(3.19 \%)$ infestation, the highest reduction of shoot $(79.65 \%)$ and fruit $(89.03 \%)$ infestation to compare with control. The sex pheromone confused the male adult for mating and thus preventing fertilized egg production vis-à-vis reduces larval and adult population build-up. These treatments altogether significantly reduced the BSFB population and its infestation level, which ultimately increased the yield of brinjal.

Alam et al., (2003) aslo reported that, the marketable fruit yield was greater in pheromone treated plots than in check plots. Use of sanitation may have contributed to the reduction in pest damage in pheromoneinstalled plots. However the experiment was done in the open field in a predominantly eggplant growing region, where migration of pest adults from elsewhere into experimental areas could not be prevented. As a result, as observed elsewhere with barrier net studies, contribution of sanitation only treatment in reducing pest damage was expected to be minimal. In addition to the commercial available pheromone water locally made waste can trap also found as effective as that of water trap, hence farmer can use this trap along with commercially available lures either for monitoring of mass trapping of shoot and fruit borer in brinjal ecosystem.

\section{References}

Alam, S.N., Rashid, M.A., Rouf, F.M.A., Jhala, R.C., Patel, J.R., Satpathy, S., Shivalingaswamy, T.M., Rai, S., Wahundeniya, I., Cork, A., Ammaranan, C., and Talekar, N.S. 2003. Development of an integrated pest management strategy for eggplant fruit and shoot borer in South Asia, 
Tech. Bull., TB28, AVRDC - The World Vegetable Center, Shanhua, Taiwan, 66 PP.

Andagopal, V.N., Prasad, T.V., Jethwa Dharmrajsinh, Gedia, M.V., Koradia, V.G. and Patel, H.V. 2011. Evaluation of pheromone traps against brinjal fruit and shoot borer, Leucinodes orbonalis Guenee. Indian J. Entomol., 72(1): 710.

Anonymous. 2014a. National Horticulture Board, Ministry of Agriculture, Government of India 85, Institutional Area, Sector-18, Gurgaon-122 015, India.

Anonymous. 2014b. Package of practice for horticultural crops, Univ. Horti. Sci., Bagalkot. pp 65-66.

Chakraborthi, S. 2001. A biorational approach for the management of brinjal fruit and shoot borer, Leucinodes orbonalis Guenee. J. Entomol. Res., 25: 73-76.

Dhandapani, N., Shelkar, U.R. and Murugan, M. 2003. Bio-intensive pest management in major vegetable crops: An Indian perspective. J. Food, Agri. Environ., 1(2): 330-339.

Kalloo, G. 1988. Biochemical basis of insect resistance in vegetables. Vegetable Breeding (Vol. II), CRC Press Inc Boca Raton, Florida. 520-570.

Rahman, M.M., Ali, M.R. and Hossain, M.S. 2009. Evaluation of combined management options for managing brinjal shoot and fruit borer. Academic J. Entomol., 2(2): 92-98.

Rajneesh, H. 2006. Management of shoot borer, Leucinodes orbonalis Guene on potato crop. M. Sc(Agri) Thesis, University of Agricultural Sciences. Dharwad (India).

Ram Prasad Mainali. 2014. Biology and management of eggplant fruit and shoot borer, Leucinodes orbonalis Guenee (Lepidoptera: Pyralidae): A review. Int. J. Appl. Sci. Biotechnol., 2(1): 18-28.

\section{How to cite this article:}

Aravinda, M., S.S. Udikeri and Karabhantanal, S.S. 2017. Evaluation of Sex Pheromone Trap Models for Monitoring of Shoot and Fruit Borer, Leucinodes orbonalis Gueene. in Brinjal. Int.J.Curr.Microbiol.App.Sci. 6(2): 575-585. doi: http://dx.doi.org/10.20546/ijcmas.2017.602.065 